\title{
Analyse Du Rebut Des Cliches Radiographiques Dans Le Service De Radiologie Et Imagerie Médicale Du Chu Campus De Lomé Au Togo
}

\author{
P. Gbande \\ L. Sonhaye \\ K. Adambounou \\ K. Lambon
}

Service de Radiologie et Imagerie Médicale CHU Campus de Lomé, Togo

B. N'timon

M. Dagbe

Service De Radiologie Et Imagerie Médicale CHU Kara, Togo

L.K. Agoda-Koussema

K.V. Adjenou

K. N'dakena

Service De Radiologie Et Imagerie Médicale CHU de Lomé, Togo

Doi: 10.19044/esj.2017.v13n33p244 URL:http://dx.doi.org/10.19044/esj.2017.v13n33p244

Abstract

Purpose: To analyze the waste factors of rejected X-rays films. Methodology: Descriptive and analytical prospective study from 1 January to 30 June 2017 carried out in the department of radiology and medical imaging of the Campus University Hospital of Lomé in Togo. Results: 4912 patients had received 5630 radiographic incidences, including 3288 (58.4\%) on the analogy and $2342(41.5 \%)$ on the digital. The reject rate was $12.5 \%$. The vast majority of the X-rays films, $682(96.9 \%)$ were rejected by the radiographers themselves just after development. The resumption frequency ranged from one repeat (550 X-rays films, or $78 \%$ ) to 4 repeats ( $8 \mathrm{X}$-rays films, or 1\%). Almost all of the rejected films, 702 (99.7\%) came from the analogical room. Chest X-ray was the incidence with more rejection in $33.9 \%$ followed by pelvic and lower limb incidences in $21 \%$ of cases. More than $2 / 3$ of the rejected films, 473 (67.2\%), came from the students' act. The causes of the rejection were mainly centering (25.5\%), underexposure (20.17\%) and overexposure (12.93). The financial loss caused by the scrap of $\mathrm{X}$-rays films amounted to about $418800 \mathrm{~F}$ CFA or $638.5 €$. Conclusion: Strengthening communication between radiographers and radiologists is necessary to avoid unnecessary repeats of patient's radiographs. 
Keywords: Rejected X-rays films, radiography, image quality, radioprotection, Togo

\section{Résume}

But : Analyser les facteurs de mise au rebut des clichés radiographiques. Méthodologie: Etude prospective descriptive et analytique allant du $1^{\text {er }}$ janvier au 30 juin 2017 réalisée dans le service de radiologie et imagerie médicale du Centre Hospitalier Universitaire Campus de Lomé au Togo. Résultats: 4912 patients avaient bénéficié de 5630 incidences radiographiques dont $3288(58,4 \%)$ à l'analogie et $2342(41,6 \%)$ au numérique. Le taux de rebut était de $12,5 \%$. 682 clichés $(96,9 \%)$ ont été rejetés par les réalisateurs eux-mêmes juste après le développement. Les clichés ont été repris une fois dans $78 \%$ et 4 fois dans $1 \%$. . Les clichés rejetés provenaient de la salle analogique dans $99,7 \%$ ds cas. La radiographie du thorax était l'incidence qui avait connue plus de rebut dans $33,9 \%$ des cas suivi des incidences du bassin et des membres inférieurs dans $21 \%$ des cas. Les clichés rejetés provenaient des actes des étudiants dans $67,2 \%$. Les causes du rebut étaient principalement le centrage $(25,5 \%)$, la sous-exposition $(20,17 \%)$ et la surexposition $(12,93)$. La perte financière causée par le rebut de clichés s'élevait à environ 418800F CFA soit 638,5€.

Conclusion: Le renforcement de la communication entre techniciens de radio et radiologues s'avère nécessaire pour éviter aux patients des reprises de clichés inutiles.

Mots-clés : Rebut de clichés, radiographie, qualité de l'image, radioprotection, Togo

\section{Introduction}

Le rebut de clichés radiographiques est l'ensemble des clichés rejetés pour raison de mauvaise qualité (Réné, 2006). Ce sont des clichés qui ne fournissent pas de manière fiable les informations diagnostiques correctes et par conséquent ne peuvent être utilisés. L'analyse du rebut de clichés radiographiques est une entité du contrôle de qualité en imagerie médicale (Yousef, et al., 2013). Elle permet de préciser l'origine et la cause du rejet afin de prendre des mesures appropriées pour l'amélioration de la qualité des services.

Les causes de ces rejets sont multiples, mais les plus fréquentes sont : les causes liées à la technique de réalisation et celles liées aux matériels de réalisation (Réné, 2006; Teferi, Zewdneh, Admassie, Nigatu, \& Kebeta, 2012). Certains clichés sont repris immédiatement par le technicien réalisant l'examen, d'autres par contre sont rejetés par le médecin radiologiste 
entrainant des conflits même s'ils ne sont pas extériorisés entre ces deux acteurs. Les rebuts de clichés sont responsables d'une irradiation du patient et du technicien (Agoda-Koussema, et al., 2013; Jabbari, Zeinali, \& Rahmatnezhad, 2012;). Ils constituent également une source de perte financière énorme supportée par les structures sanitaires dans le monde surtout dans les pays en voie de développement (Réné, 2006; Bingchao, 1999; Diallo, 2011).

Au Togo, aucune véritable étude sur ce sujet n'a encore été réalisée. C'est pour cela que nous avons entrepris ce travail dans le but d'analyser les facteurs de mise au rebut des films radiographiques au CHU Campus de Lomé et plus spécifiquement en identifiant les paramètres en cause dans le rejet de ces clichés radiographiques, analyser les différences dans les perceptions de la qualité des images entre techniciens et médecins radiologues et enfin, évaluer la perte financière supportée par la structure.

\section{Méthodologie}

Il s'est agi d'une étude prospective descriptive et analytique portant sur les clichés radiographiques rejetés pour leur mauvaise qualité. Elle s'est étendue du $1^{\mathrm{er}}$ janvier au 30 juin 2017 soit sur une période de 6 mois.

Notre étude a été effectuée dans le service de radiologie et imagerie médicale du CHU-Campus de Lomé au Togo. Le CHU-Campus est une formation sanitaire universitaire publique, situé dans le district $\mathrm{N}^{\circ} 5$ de Lomé. Il est implanté dans l'aire géographique de l'Université de Lomé. C'est un centre de référence du système national de soins.

Nous avons inclus dans notre étude tous les clichés rejetés pour leur mauvaise qualité quelle que soit la cause. Les examens repris parce que les initiaux n'ont pas été retrouvés, les clichés repris pour intérêt scientifique et les films qui ont servi de test après un dépannage n'ont pas été inclus dans cette étude.

La collecte des données a été faite sur la base d'une fiche d'enquête préalablement établie. Notre démarche a consisté au remplissage quotidien de ces fiches toutes les fois qu'un cliché était rejeté soit juste après le développement, soit au cours de la lecture par le médecin radiologue. Ensuite nous avons compté le nombre total de films rejetés, classé selon les formats de films, analysé les causes du rejet et évalué le coût total des films rejetés.

Les données collectées ont été analysées et traitées à l'aide du logiciel EPI info version 3.5.1.

\section{Résultats}

Durant les six mois de notre étude, 4912 patients avaient bénéficié de 5630 incidences radiographiques dont 3288 à l'analogie soit 58,4\% des clichés et 2342 au numérique soit $41,6 \%$. Le nombre total des clichés rejetés 
était de 704 soit $12,5 \%$ (Figure 1). Ces clichés rejetés provenaient de la salle analogique (702 soit $99,7 \%$ ) et seulement 2 clichés de la salle numérique.

Les réalisateurs ont rejété $682(96,9 \%)$ clichés juste après le développement. Les médecins radiologues ont rejété $22(3,1 \%)$ clichés pendant l'interprétation .

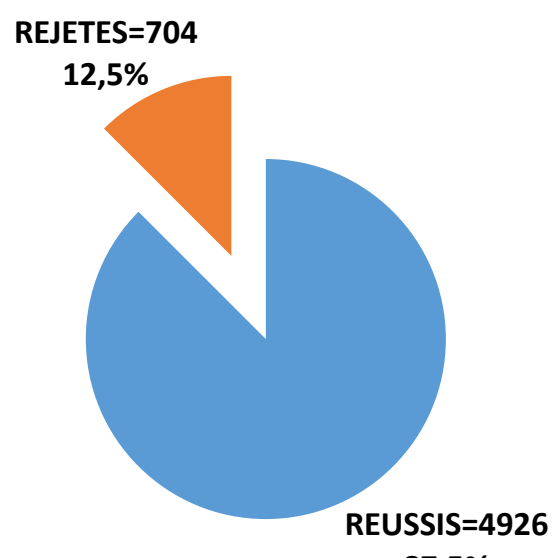

$87,5 \%$

Figure 1 : Bilan global des clichés réalisés durant l'étude

Pour la même incidence et chez le même patient, les clichés ont été repris une fois dans $78 \%$ des cas et 4 fois dans $1 \%$ des cas conformément à la figure 2 .

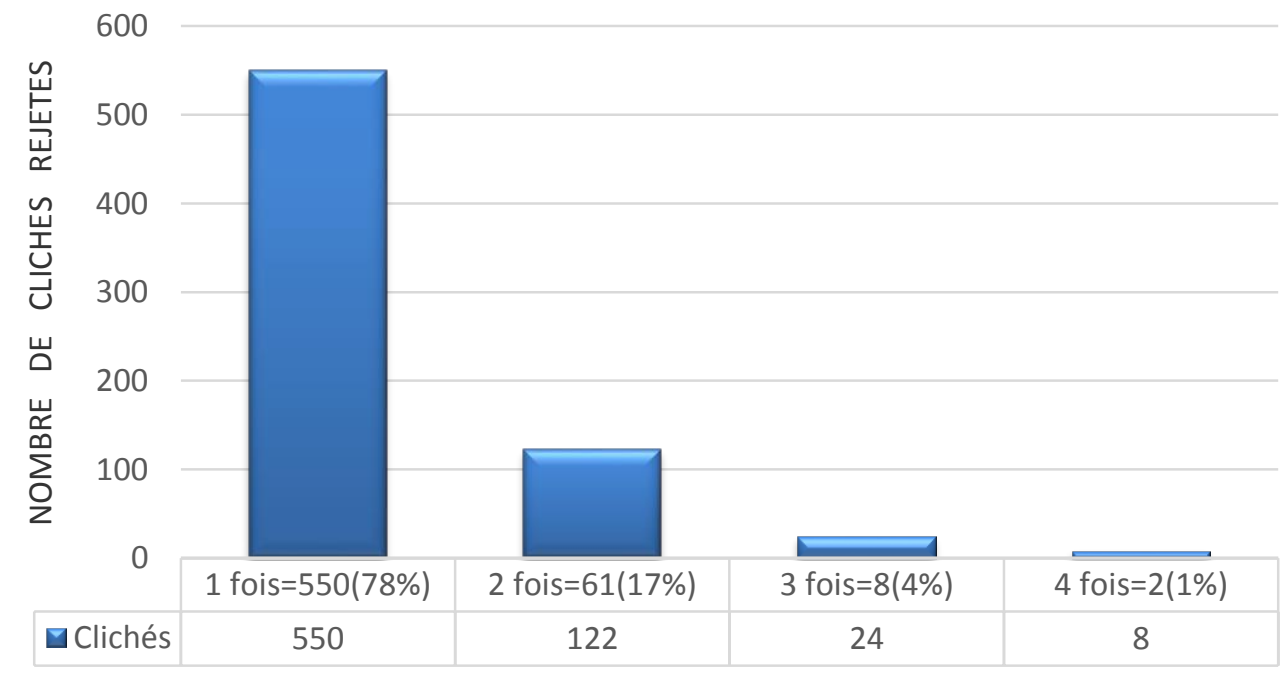

Figure 2 : Répartition des clichés rejetés en fonction du nombre de reprise

La radiographie du thorax était l'incidence qui avait connu plus de rebut dans $33,9 \%$ des cas suivi des incidences du bassin et des membres inférieurs dans $21 \%$ des cas. La radiographie de l'abdomen sans préparation 
et l'hystérosalpingographie étaient les examens qui ont connu moins de rejet (Tableau I).

Tableau I: Répartition des clichés rejetés selon le type d'examen

\begin{tabular}{ccc}
\hline & Nombre & Pourcentage \\
\hline Thorax & 239 & 33,9 \\
Bassin et membres inférieurs & 148 & 21 \\
Rachis & 107 & 15,2 \\
Crâne & 84 & 11,9 \\
Membres suéprieurs & 70 & 10 \\
ASP* & 28 & 4 \\
Hystérosalpingographie & 28 & 4 \\
Total & $\mathbf{7 0 4}$ & $\mathbf{1 0 0}$ \\
\hline
\end{tabular}

*Radiographie de l'abdomen sans préparation

Les clichés rejetés étaient des actes des étudiants stagiaires dans 67,2 $\%$ (473) des cas. La perte financière causée par le rebut de clichés s'élevait à environ 418800 F CFA (Tableau II). Les pertes indirectes: le temps, l'usure des appareils et la perte liée aux produits de développement (fixateur, révélateur et eau) n'ont pas pu être évaluées avec certitude.

Tableau II : Evaluation de la perte financière liée au rebut.

\begin{tabular}{cllcc}
\hline $\begin{array}{c}\text { Format de } \\
\text { film }\end{array}$ & $\begin{array}{c}\text { Nombre de } \\
\text { clichés }\end{array}$ & $\begin{array}{c}\text { Nombre de } \\
\text { paquets* }\end{array}$ & Prix d'un paquet & Montant (CFA) \\
\hline $35 \times 43 \mathrm{~cm}$ & 201 & 2,01 & $80000 \mathrm{~F}$ & 160800 \\
$24 \times 30 \mathrm{~cm}$ & 135 & 1,35 & $45000 \mathrm{~F}$ & 60750 \\
$20 \times 40 \mathrm{~cm}$ & 95 & 0,95 & $55000 \mathrm{~F}$ & 52250 \\
$30 \times 40 \mathrm{~cm}$ & 94 & 0,94 & $65000 \mathrm{~F}$ & 61100 \\
$18 \times 24 \mathrm{~cm}$ & 92 & 0,92 & $25000 \mathrm{~F}$ & 23000 \\
$35 \times 35 \mathrm{~cm}$ & 87 & 0,87 & $70000 \mathrm{~F}$ & 60900 \\
Total & 704 & - & - & 418800 \\
\hline
\end{tabular}

*un paquet compte 100 films.

'Parmi les causes du rebut des clichés, on notait prioritairement le centrage inexact dans $25,57 \%$ comme le montre les figures 3 et 4 . La sousexposition (clichés trop clairs) dans $20,17 \%$ des cas et l'insuffisance des critères de réussite du cliché (Figure 5) dans 14,35\%. Les clichés surexposés (trop sombres) avaient représenté $12,93 \%$ des rejets (Tableau III). 
Tableau III : Répartition globale des clichés rejetés en fonction de la cause

\begin{tabular}{ccc}
\hline & Nombre & Pourcentage \\
\hline Centrage inexact & 180 & 25,57 \\
Cliché sous exposé & 142 & 20,17 \\
Insuffisance critères de réussite & 101 & 14,35 \\
Cliché surexposé & 91 & 12.93 \\
Anomalie du tube & 48 & 6,82 \\
Flou cinétique (Mouvement du patient) & 45 & 6,40 \\
Fuite de la lumière (voile) & 41 & 5,83 \\
Mauvaise incidence & 12 & 1,70 \\
Corps étrangers (mauvaise préparation du patient) & 11 & 1,56 \\
Erreur au développement du film & 10 & 1,42 \\
Cliché mal orienté & 02 & 0,28 \\
Le non-fonctionnement de la grille & 01 & 0,14 \\
Superposition d'images & 01 & 0,14 \\
Rayure en bas du cliché & 01 & 0,14 \\
Aaunissement du film par défaut de rinçage & 01 & 0,14 \\
Autres* & 17 & 2,41 \\
Total & 704 & 100 \\
\hline
\end{tabular}

*main de l'accompagnant sur l'image ; canule hors canal cervical et insuffisance d'opacification tubaire (pour les hystérosalpingographies)

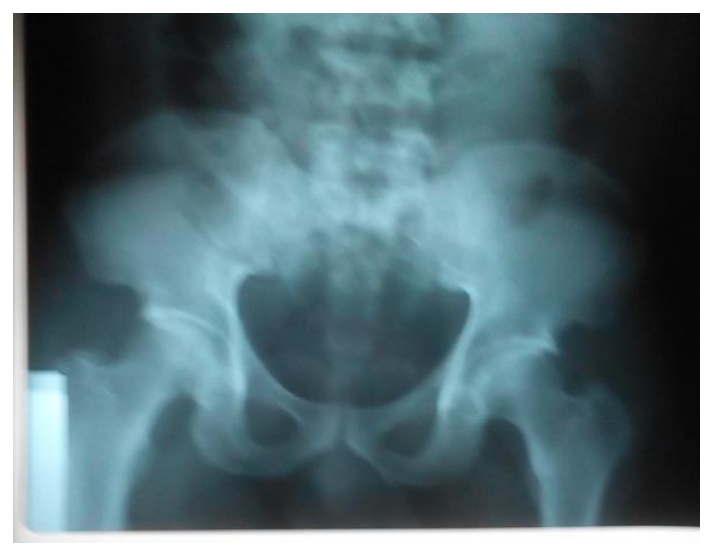

Figure 3 : Radiographie du bassin de face, cliché excentré du au mauvais positionnement du patient

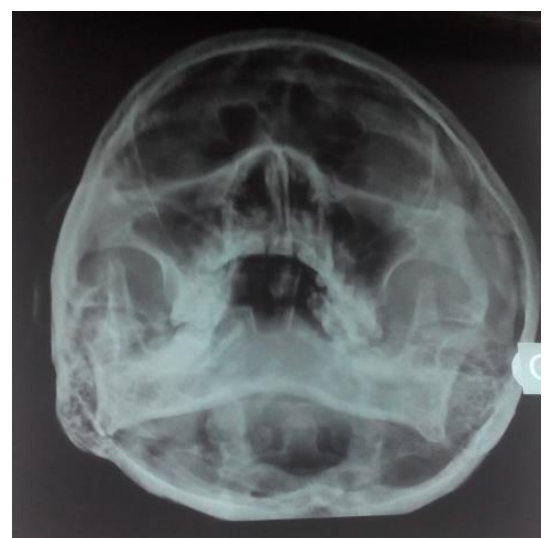

Figure 5: Cliché du Blondeau non symétrique, pas de symétrie des os malaires 


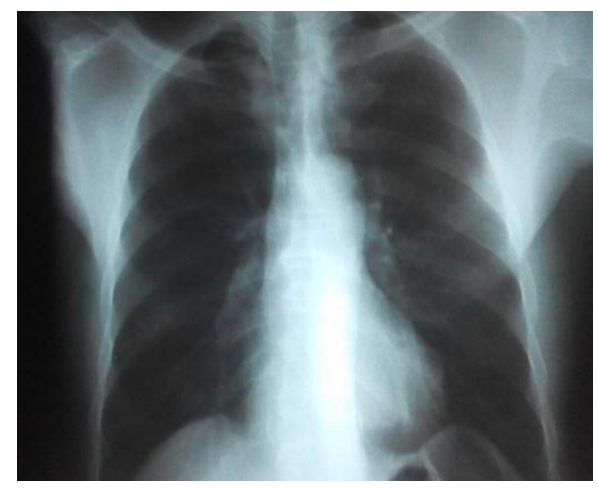

Figure 4: Radiographie du thorax demandée dans le cadre d'un bilan HTA et rejetée pour cul-de-sac costo-diaphragmatiques rasés, défaut

Discussion de centrage.

\section{Méthodologie}

Cette étude, menée grâce aux fiches préalablement établies avait porté sur l'analyse prospective de 704 clichés de radiographie rejetés pour leur mauvaise qualité. Elle a été réalisée dans le service de radiologie du CHU Campus de Lomé sur une période de 6 mois. Cette étude pouvait être étendue sur plusieurs structures sanitaires et même sur une période beaucoup plus longue afin d'obtenir des résultats beaucoup plus objectifs et plus réalistes. Toutefois nous estimons que cela n'enlève en rien la validité de ces résultats.

\section{Bilan général des clichés rejetés}

Le taux global de rejet de clichés était de 12,5\%. Agoda-Koussema et al avaient trouvé 14\% en pratique radio pédiatrique (Agoda-Koussema, et al., 2013). Au Nigéria, Abubakar et al avaient rapporté un taux beaucoup plus élevé avec 26,04\% (Abubakar et al., 2016). Par contre Daniel Zewdeneh en Ethiopie avait retrouvé un taux de rejet de 4,94\% (Daniel, Seife, \& Daniel, 2008). De même Mottola Virginie et Dubuis Carole en Suisse avaient retrouvé un taux de $2,9 \%$ en mammographie de dépistage (Mottola \& Dubuis, 2011). Le taux élevé de rebut de clichés dans notre étude pourrait s'expliquer par le fait que le CHU Campus de Lomé est un hôpital universitaire où on retrouve beaucoup d'étudiants stagiaires qui ne maitrise pas assez bien les bonnes méthodes pratiques. Pour certains il s'agit de leur première manipulation d'une table de radiologie.

La numérisation est le processus par lequel un signal analogique est transformé en un signal numérique. Elle a plusieurs avantages en imagerie médicale parmi lesquels on note l'économie des films radiographiques. 
C'est un aspect très observé en pratique radiologique (Hoogewoud \& Girard, 2001; Baudhuin, Nuzzo, \& Zimmermann, 2004). Dans notre étude, c'est seulement $0,3 \%$ des clichés rejetés qui provenaient de la salle de radiographie numérique.

Pour la même incidence et chez le même patient, on pouvait répéter jusqu'à 4 fois avant d'obtenir un bon résultat. L'irradiation excessive provient en majorité de la répétition des clichés chez les patients et ne fait qu'augmenter la dose d'irradiation du patient et du personnel.

La radiographie du thorax était l'incidence qui avait connu plus de rebut dans 33,9\%, même constat fait dans plusieurs études antérieures (Daniel, Seife, \& Daniel, 2008; Abubakar, et al., 2016; Tchaou, et al., 2016). Ce classement en tête de la radiographie du thorax est lié a priori à la fréquence plus élevée de demande des radiographies du thorax dans le monde (Vishal, et al., 2011; Adambounou, et al., 2013) que par une complexité technique de sa réalisation.

\section{Les causes du rebut des clichés et évaluation de la perte financière}

Parmi les causes du rebut, on notait principalement, le centrage inexact dans plus du quart des clichés, la sous-exposition (clichés trop clairs) et la surexposition (trop sombres). Ces facteurs ont été mis en cause dans plusieurs études antérieures (Jabbari, Zeinali, \& Rahmatnezhad, 2012; Abubakar, et al., 2016; Sniureviciute \& Adliene, 2005). Ils ont été mis en cause dans plus de la moitié des rejets. Il a été démontré que ces facteurs sont liés à l'erreur humaine c'est-à-dire, celle du technicien réalisateur.

Dans cette étude, la grande majorité des clichés soit $96,9 \%$ ont été rejetés par les réalisateurs eux-mêmes après le développement et $3,1 \%$ des clichés jugés bons par les réalisateurs avaient été rejetés par les médecins radiologues pendant l'interprétation. Cette différence de perception entre techniciens de radio et médecins radiologues concernant la qualité de l'image radiographique contredit le deuxième principe de la radioprotection qui est l'optimisation. Les critères d'évaluation de la qualité d'une image radiographique devraient être les mêmes aussi bien pour le technicien que le radiologue. Un bon cliché radiographique est celui qui présente toutes les informations nécessaires pour répondre à la question du demandeur. Une image radiographique de mauvaise qualité pour le technicien, peut contenir toutes les informations que cherche le radiologue pour poser son diagnostic et donc ne nécessiterait peut être pas de reprise (Figure 4). Cela suggère qu'il y'a besoin d'améliorer la communication entre les radiologues et les techniciens de radio pour la réduction des reprises inutiles de clichés.

La perte financière causée par le rebut de clichés s'élevait à environ 418800F CFA soit environ 638,5 € en 6 mois. Cette évaluation n'a pris en compte que le prix des formats de films concernés. Une évaluation un peu 
plus objective, prenant en compte les autres paramètres mis en jeu trouverait une perte un peu plus élevée. Cette perte est celle qui est supportée par le service de radiologie. Notons que le rebut de clichés entraine aussi de pertes pour le patient: allongement du temps de réalisation, nécessité de revenir pour la reprise de l'examen.

\section{Conclusion}

Etude prospective analytique portant sur les clichés radiographiques rejetés pour leur mauvaise qualité, elle nous a permis de nous rendre compte que l'erreur technique est la principale cause de rejet. Elle nous a permis aussi de savoir qu'il existe une différence de perception de la qualité de l'image radiographique entre techniciens de radio et radiologues. La nécessité de renforcer la communication entre ces deux acteurs s'avère nécessaire pour éviter aux patients des reprises inutiles.

\section{References:}

1. Abubakar A., Isma'il Gana M., Gunda N., Nwobi I., Yusuf M., Luntsi G., \& al. (2016). Assessment of X-ray Film Reject Rate in Two Tertiary Healthcare Institutions in Northeastern Nigeria. The IJST, 4(8), 53-56.

2. Adambounou K., Adjenou K V., Gbande P., Agoda-Koussema LK., Jonhson K., Dagbé M. et al. (2013). Profil et évaluation de la justification des examens radiographiques standards réalisés dans les CHU de Lomé( TOGO). J Afr Imag Méd, 5(03), 148-158.

3. Agoda-Koussema LK., Koffi K., Adjenou KV., Afansounoudji A., Amouzou K., Awobanou K., \& al. (2013). La pratique de la radiologie conventionnelle au Togo: A propos de 580 cas colligés aux chu de Lomé. J Afr Imag Med, 5(1), 75-8.

4. Baudhuin P., Nuzzo V., \& Zimmermann N. (2004). Digital imaging: conventional digital imaging! J Radiol, 85(7-8), 1037-45.

5. Bingchao Z. (1999). Etude de la mise au rebut des clichés radiographiques à l'hôpital national de Kati. Med Afr Noire, 46(10), 479-481.

6. Daniel Z., Seife T., \& Daniel A. (2008). X-ray reject analysis in Tikur Anbessa and Bethzatha hospitals. Ethiop J Health Dev, 22(1), 63-67.

7. Diallo L. (2011). Analyse du rejet des films radiologiques dans le centre médical CELY de Bamako avant la mise en oeuvre de la numérisation. Bamako, Mali: Faculté de Médecine de Pharmacie et d'Odonto-Stomatologie.

8. Hoogewoud H., \& Girard J. (2001). La révolution numérique en radiologie. Rev Méd Sui Rom, 21(8), 591-4. 
9. Jabbari N., Zeinali A., \& Rahmatnezhad L. (2012;). Patient dose from radiographic rejects/repeats in radiology centers of Urmia University of Medical Sciences, Iran. Health, 4(2), 94-100. Récupéré sur http://dx.doi.org/10.4236/health.2012.42015.

10. Mottola V., \& Dubuis C. (2011). Analyse de rejet en mammographie : comparaison diagnostique/dépistage. Suisse Occidentale: Haute école cantonale vaudoise de la santé.

11. Réné K. (2006). Etude de la mise au rebut des films radiographiques dans le service de radiologie et de médecine nucléaire de l'hopital du point G. Bamako: FMPOS.

12. Sniureviciute M., \& Adliene D. (2005). Problems with film processing in medical X-Ray imaging in Lithuania. Radiation Protection Dosimetry, 5, 260-263(5), 260-263.

13. Tchaou M., Gnakadja G., N'timon B., Sonhaye L., Amadou A., Dansou M., \& al. (2016). Revue des doses d'exposition et la justifications des radiographies standards en pratique pédiatrique au Togo. European Scientific Journal, 12(24), 223-233.

14. Teferi S., Zewdneh D., Admassie D., Nigatu B., \& Kebeta K. (2012). $\mathrm{X}$-ray film reject rate analysis at eight selected government hospitals in Addis Ababa, Ethiopia, 2010. Ethiop J Health Dev, 26(1), 54-9.

15. Vishal V., Viswanath V., Praveen J., Sasikanth N., Ayanava M., Arjomand F., al. (2011). The utility of routine admission chest X-ray films on patient care. European Journal of Internal Medicine, 22(3), 286-288.

16. Yousef M., Edward C., Ahmed H., Bushara L., Hamdan A., \& Helnaiem N. (2013, january-june). Film Reject Analysis For Conventional Radiography In Khartoum Hospitals. Asian Journal of Medical Radiological Research, 1(1), 34-8. 